\title{
Patency period of a metallic ureteral stent and its determinants in patients with malignant ureteral obstruction: a prospective review
}

\author{
Yasuyuki Kobayashi* (D, Hiroki Arai and Masahito Honda
}

\begin{abstract}
Background: Malignant ureteral obstruction caused by extrinsic compression of a primary malignant tumour or by metastatic disease is an indicator of poor prognosis with a median life expectancy of about one year. We examined clinical outcomes following Resonance Metallic Ureteral Stent (Cook Medical, Bloomington, IN) placement in patients with malignant ureteral obstruction.

Methods: This was a prospective study of patients with malignant ureteral obstruction who underwent Resonance Metallic Ureteral Stent placement from April 2016 to March 2021. We registered 21 patients (27 collecting systems) with malignant ureteral obstruction and observed them prospectively. The patients first underwent polymer ureteral stent placement followed by replacement with a metallic ureteral stent one month later. Primary outcome was the metallic ureteral stent patency period based on both serum creatinine and the level of hydronephrosis; secondary outcomes were factors affecting patency period and stent-related complications such as symptoms of obstruction (flank pain), bladder irritation, haematuria, and urinary tract infection (presence or absence of fever).
\end{abstract}

Results: The study comprised 21 patients (six men, 15 women) with a mean age of 72 years. The median stent patency period in days was not available (NA) $(95 \% \mathrm{Cl} 210-\mathrm{NA})$ due to the inability to extract this value from the Kaplan-Meier curve because the event rate did not reach 50\%, and the one-year patency rate was $59.2 \%$ (95\% Cl 23.2-82.9). A normal serum creatinine (0.65 to $1.07 \mathrm{mg} / \mathrm{dL}$ for men and 0.46 to $0.79 \mathrm{mg} / \mathrm{dL}$ for women) one week after polymer ureteral stent placement was a significant factor affecting the long-term metallic ureteral stent patency period. There were no major complications.

Conclusion: The Resonance Metallic Ureteral Stent was effective and safe for patients with malignant ureteral obstruction. A normal serum creatinine level one week after placement of a polymer ureteral stent may predict a longer patency period of metallic ureteral stents in patients with malignant ureteral obstruction.

Keywords: Malignant ureteral obstruction, Metallic ureteral stent, Patency period, Resonance Metallic Ureteral Stent, Stent obstruction

\section{Background}

Malignant ureteral obstruction (MUO), which is historically confirmed as upper urinary tract obstruction with hydronephrosis resulting from malignant disease, is

*Correspondence: ya_su_koba@yahoo.co.jp

Department of Urology, Kinki Central Hospital of Mutual Aid Association of Public School Teachers, 3-1 Kurumazuka, Itami, Hyogo 664-8533, Japan caused by extrinsic compression from a primary malignant tumour or by metastatic disease [1]. MUO is an indicator of poor prognosis, and the median life expectancy is about one year [2]. Patients with MUO should be treated to prevent renal failure, flank pain, and urinary tract infection (UTI) [3, 4]. The final treatment goals are to preserve renal function, especially in patients requiring further chemotherapy, and to prevent localised pain 
and UTI to help maintain quality of life (QOL). Many types of polymer ureteral stents are available to relieve obstruction as an initial approach, but they have a high rate of obstruction and require periodic exchange every three to six months $[5,6]$. Nephrostomy can also be used to relive obstruction because of its high success rate, but it tends to deteriorate patient QOL because of the external drainage tube and its risk of dislodgment [7].

The Resonance Metallic Ureteral Stent (Cook Medical, Bloomington, IN, USA), a completely unfenestrated, coiled, all-metallic double-pigtail ureteral stent, has been used as an efficacious alternative. The metallic ureteral stent is highly resistant to extrinsic compression compared with polymer ureteral stents and has a longer indwelling time of up to 12 months [8]. As the stent has no side ports, urine drainage is maintained by urine dripping into its inner lumen or around its coils and by drainage along the outside of the stent, as with the polymer ureteral stent [9].

Although there are several reports of obstruction rates and factors related to metallic ureteral stents, there are few retrospective studies on the patency period and factors affecting this period in patients with MUO [4, $10,11]$. One recent prospective observational study on metallic ureteral stents unfortunately did not report the precise patency period or factors affecting the period in patients with MUO [12]. We aimed to determine which characteristics of these patients lead to longer patency periods. Therefore, we conducted a single-centre prospective study to examine the patency period of metallic ureteral stents and its determinants in 21 patients with MUO.

\section{Methods}

This was a prospective study of patients with MUO who underwent Resonance Metallic Ureteral Stent placement from April 2016 to March 2021. The ethics committee of our institution approved this prospective study on 18 January 2016 (approval no. 276), 27 October 2018 (approval no. 364), and 18 June (approval no. 421). This study complied with the international ethical recommendations of the Declaration of Helsinki [13]. Written informed consent was obtained from all patients studied. The authors confirm the availability of, and access to, all original data reported in this study.

A flow chart of the present study is shown in Fig. 1. We consider the metallic ureteral stent to be standard care in patients with MUO in our institution and proceed with therapy according to the flow chart in Fig. 2 . Eligible patients were aged 20 years or older and had not undergone previous therapy for MUO. We performed stent placement in all patients with MUO except those with unilateral mild hydronephrosis, no deterioration of renal function, no flank pain, and no infection. We excluded patients with primary urinary tract (pelvis, ureter, and bladder) cancer and prostate cancer. Between April 2016 and March 2021, we registered 21 patients with MUO referred to our outpatient department. We treated bilateral ureters in the same patient as individual ureters, following previous studies $[4,10]$. Patients had upper urinary tract obstruction with hydronephrosis as revealed by computed tomography. We performed stent placement in 21 patients, bilaterally in six and unilaterally in 15 patients. We classified the cause of MUO as a solid mass (direct compression by tumour or lymph node dissemination) or peritoneal metastasis.

The primary outcome was the patency period of the metallic ureteral stent based on both serum creatinine and the level of hydronephrosis. The secondary outcomes were factors affecting the patency period of the metallic ureteral stent and stent-related complications such as symptoms of obstruction (flank pain), bladder irritation, haematuria, and UTI (presence or absence of fever). We defined stent obstruction based on not only deterioration of renal function as assessed by serum creatinine but also progression of hydronephrosis considering ultrasonography results during follow-up. We assessed hydronephrosis according to the Classification of the Japanese Society of Pediatric Urology as defined in Table 2 [14]. We defined the patency period as the time from the day of initial metallic ureteral stent placement to the day of stent obstruction or last follow-up without obstruction. We did not consider stent migration with sufficient urine drainage to be stent obstruction and calculated the patency period until the day of stent removal in this case. We defined overall survival as the time from first visit to our outpatient department to death.

We performed retrograde pyelography at initial stent placement to confirm the ureteral anatomy (including ureter length by direct measurement, stricture position, and length). Referring to a previous study [15], we measured ureter length with a 5-Fr 70-cm ruled ureteral catheter (Flexima Ureteral Catheter Open End, Boston Scientific Corporation, Spencer, IN, USA). We then inserted a 6-Fr multi-length $(22-32 \mathrm{~cm})$ polymer ureteral stent (INLAY OPTIMA; C.R. Bard, Inc. Covington, IN, USA) per the manufacturer's recommendation. We performed follow-up at one week and one month after stent placement.

One month after placement of the polymer ureteral stent, we replaced it with a metallic ureteral stent (Fig. 3). We performed retrograde pyelography again if re-evaluation was considered necessary, such as for a tortuous ureter noticed at the initial stent placement. We inserted the 8.3-Fr metallic ureteral stent introducer over the guidewire under fluoroscopic guidance and advanced it to the 


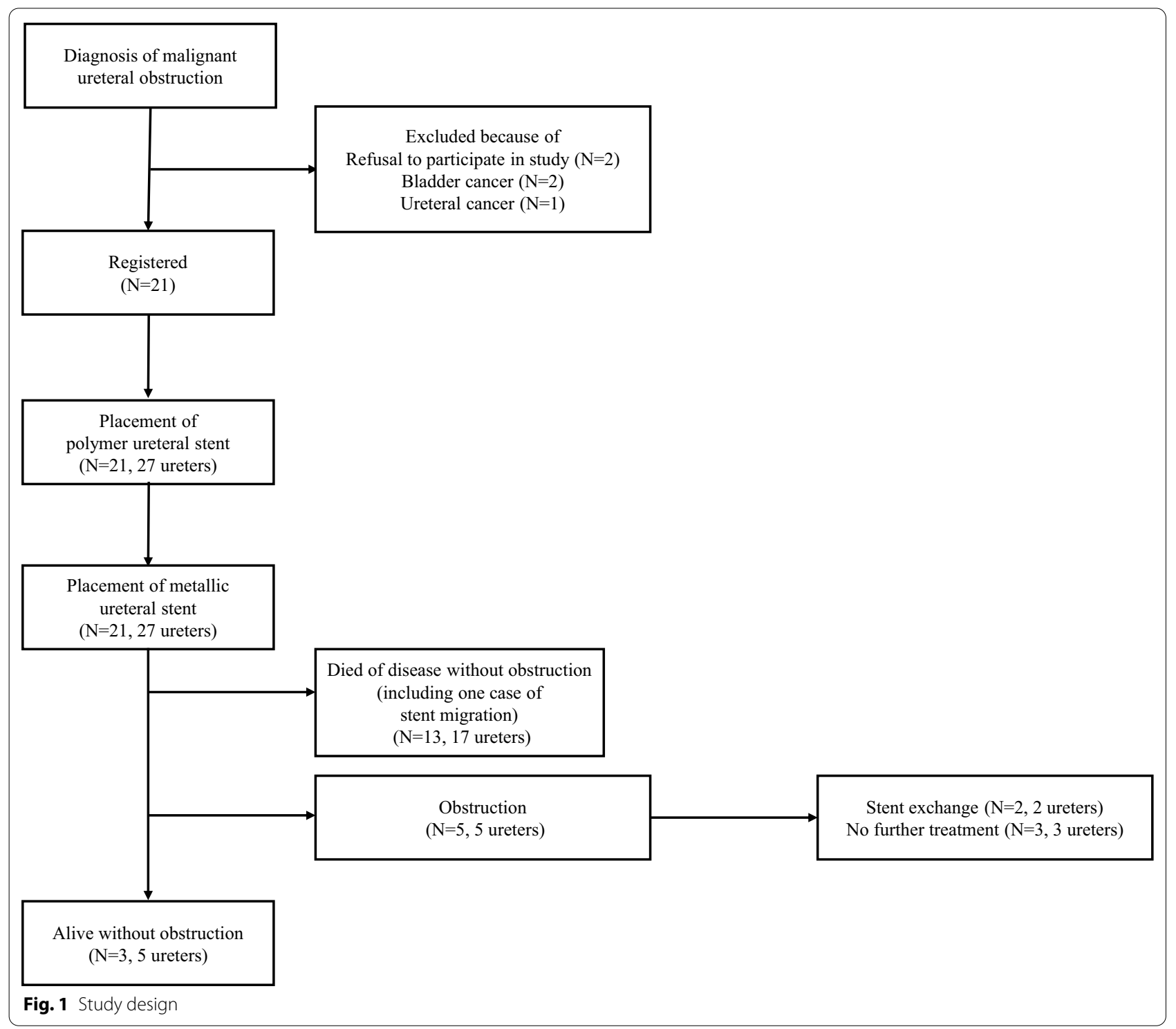

ureteropelvic junction. We confirmed that the proximal curl of the stent was located in the renal pelvis and the distal curl was located in the bladder. The length of the metallic ureteral stent was determined according to the length of the ureter. We did not perform balloon dilation of ureteral strictures. We performed follow-up at one week and one month after stent placement.

Thereafter, we performed follow-up every month until death. In case of metallic ureteral stent obstruction, we planned to exchange the stent, perform a nephrostomy, or provide best supportive care according to the patient's wishes. All patients were instructed to visit the emergency department if experiencing symptoms such as flank pain, fever, remarkable macroscopic haematuria, and voiding disorders.
The patency period and overall survival were calculated using the Kaplan-Meier method. The log-rank test was used to evaluate the relationship between the patency period and causes of ureteral obstruction (solid mass or peritoneal metastasis), side of obstruction (unilateral or bilateral), UTI (with or without after placement of a metallic ureteral stent), normal serum creatinine one week after polymer ureteral stent placement, patient age ( $<70$ years old or not), performance status ( 0 or not), and serum creatinine before metallic ureteral stent placement $(<2 \mathrm{mg} / \mathrm{dL}$ or not). All P-values were two-sided, and a value of $<0.05$ was considered statistically significant. All statistical analyses were performed with open-source software EZR version 1.27 (Saitama Medical Center, Jichi Medical University, Saitama, Japan), which is a graphical 
Diagnosis of malignant ureteral obstruction

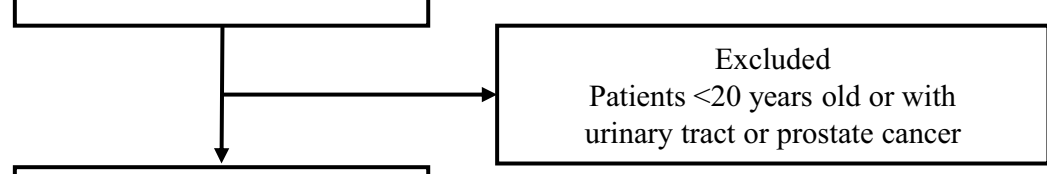

Placement of polymer ureteral stent

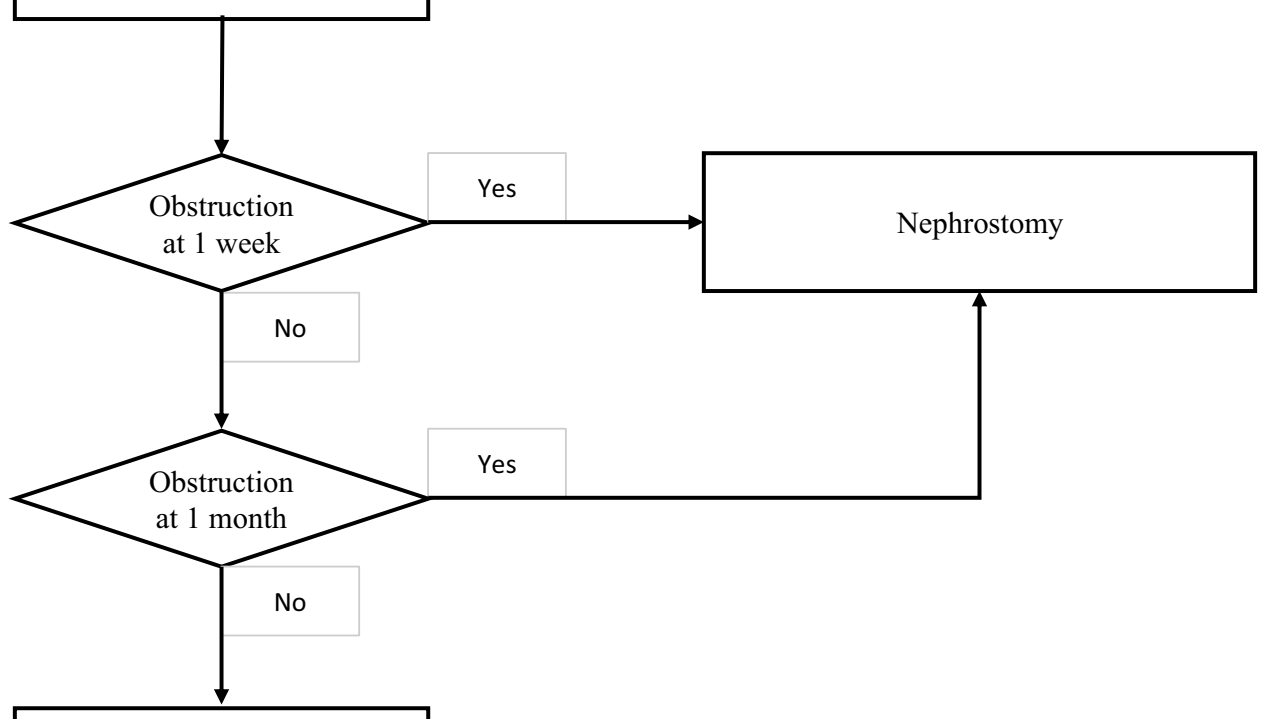

Placement of metallic ureteral stent

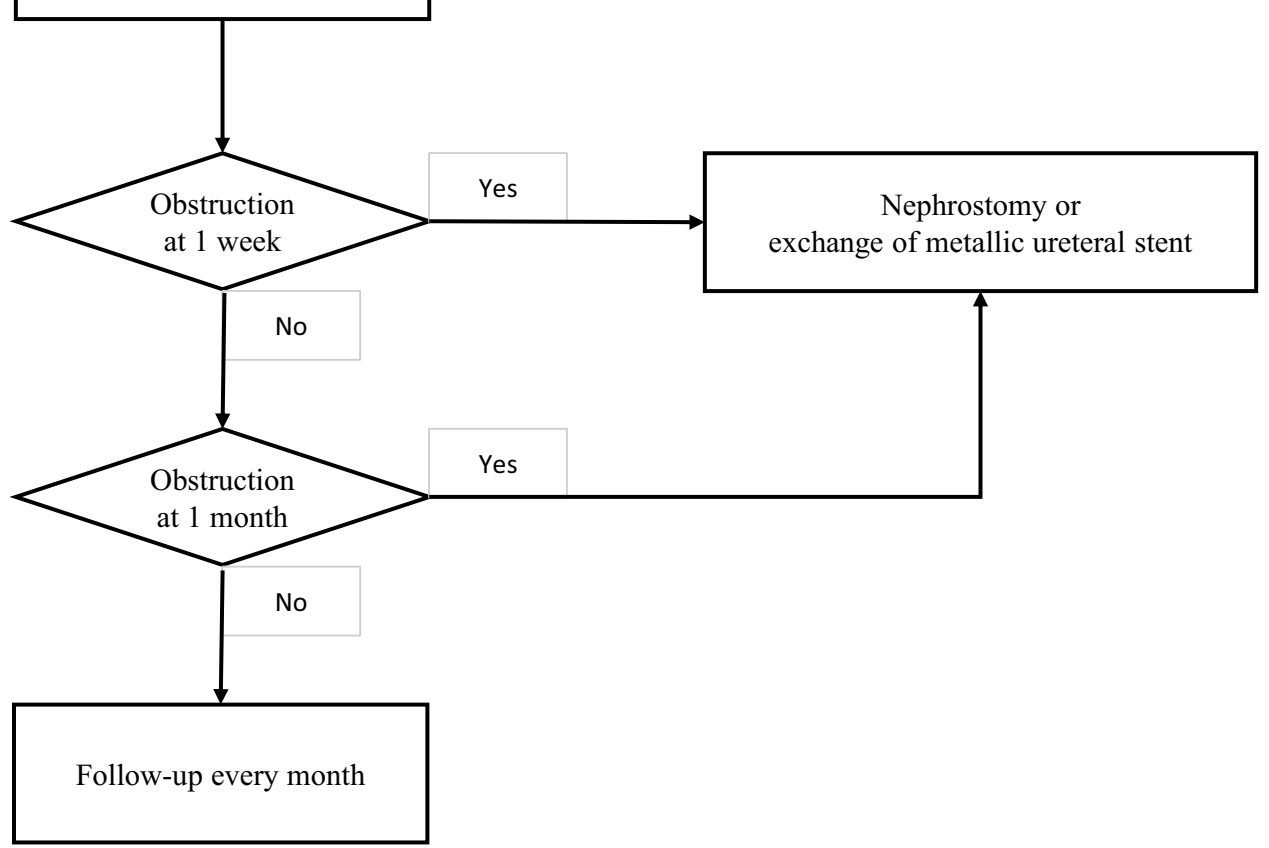

Fig. 2 Flow chart of patients' clinical outcomes 


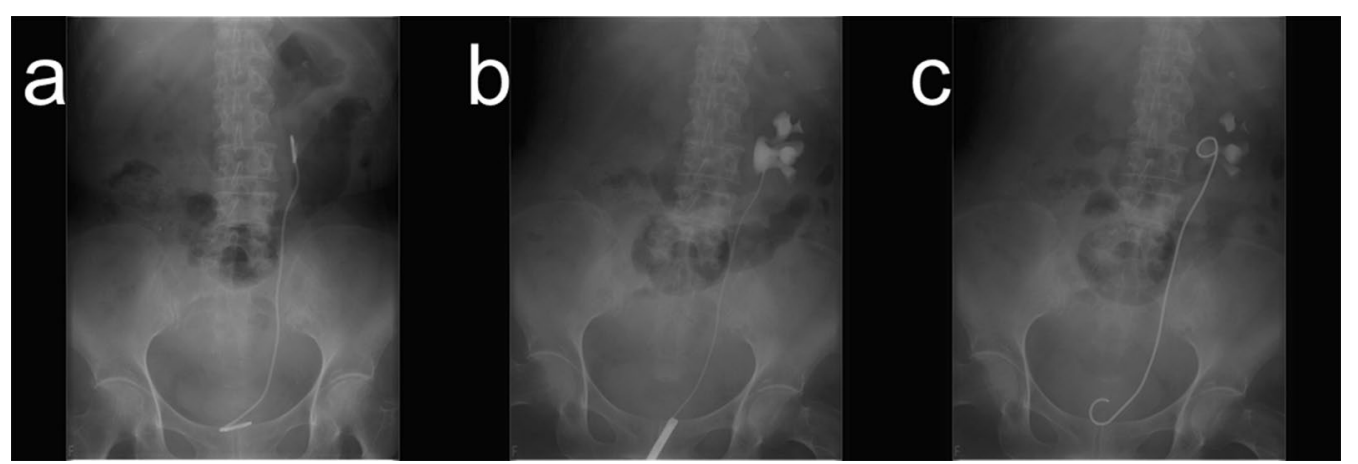

Fig. 3 a One month after placement of the polymer ureteral stent. $\mathbf{b}$ We performed retrograde pyelography again if re-evaluation was considered necessary, such as for a tortuous ureter noticed at the initial stent placement. $\mathbf{c}$ We confirmed that the proximal curl of the stent was located in the renal pelvis, and the distal curl was located in the bladder

user interface for $\mathrm{R}$ (The $\mathrm{R}$ foundation for Statistical Computing, Vienna, Austria). We analysed the data as of 17 June 2021.

\section{Results}

We registered 21 patients in the present study and a placed metallic ureteral stent in 27 collecting systems. The characteristics of the 21 patients (six men, 15 women) are listed in Table 1 . None of the patients received radiotherapy before enrolment.

In the case of obstruction of either the polymer or metallic ureteral stent at one week or one month after stent placement, we planned to perform a nephrostomy, but no obstruction occurred in any of the patients. Changes in the serum creatinine level and hydronephrosis before and one week after placement of a polymer ureteral stent, and one week after placement of the metallic ureteral stent, are shown in Table 2. The median serum creatinine level (range) at each stage was 1.04 (0.58-5.27), 0.86 (0.5-3.79), and $0.99(0.6-3.89) \mathrm{mg} / \mathrm{dL}$, respectively, and did not change significantly. All patients obtained relief of hydronephrosis after placement of the polymer stent and maintained relief after placement of the metallic stent.

The complications associated with the metallic ureteral stent are also shown in Table 2. Among those with bladder irritation, three patients had mild bladder irritation one month after placement of the metallic ureteral stent that resolved spontaneously. The remaining two patients experienced bladder irritation at two and six months, respectively, after stent placement that was resolved with the administration of solifenacin succinate. The patient with macroscopic haematuria had mild haematuria after placement of the metallic stent that resolved spontaneously. UTI resolved in all patients after the administration of oral antibiotics. The patient with stent migration had no subjective symptoms when the proximal curl of the stent moved to the upper ureter, and no progression of hydronephrosis or deterioration of renal function occurred. We exchanged the metallic ureteral stent according to the patient's wish, and migration never recurred. No major complications or visits to the emergency room occurred as a result of the stent-related complications.

The clinical outcomes of the patients are shown in Fig. 4. Obstruction occurred in five patients, of whom two easily underwent stent exchange. The patient in case 5 experienced disease progression and stent obstruction after chemotherapy withdrawal. Although she resumed chemotherapy after stent exchange, she was eventually diagnosed as having progressive disease and stent obstruction occurred thereafter. One patient did not require stent exchange due to best supportive care. The other two patients were in poor general condition and did not require additional treatment. The extracted metallic ureteral stents did not display apparent encrustation macroscopically. Three patients were still alive without obstruction at the time of data analysis. The median overall survival time was 209 days $(95 \%$ confidence interval [CI] 92-389), and the one-year survival rate was $32.6 \%$ (95\% CI 13.1-53.8) (Fig. 5a). The median stent patency period in days was not available (NA) (95\% CI 210-NA), and the 1-year patency rate was 59.2\% (95\% CI 23.2-82.9) (Fig. 5b). We did not find significant differences in either the stent patency period between patients with direct compression by solid mass versus peritoneal metastasis (Fig. $5 \mathrm{c}$ ), that between patients with unilateral obstruction versus bilateral obstruction (Fig. 5d), or that between patients with or without UTI after placement of the metallic ureteral stent (Fig. 5e). However, there was a significant difference in the patency period between patients with a normal creatinine one week after placement of the polymer ureteral stent 


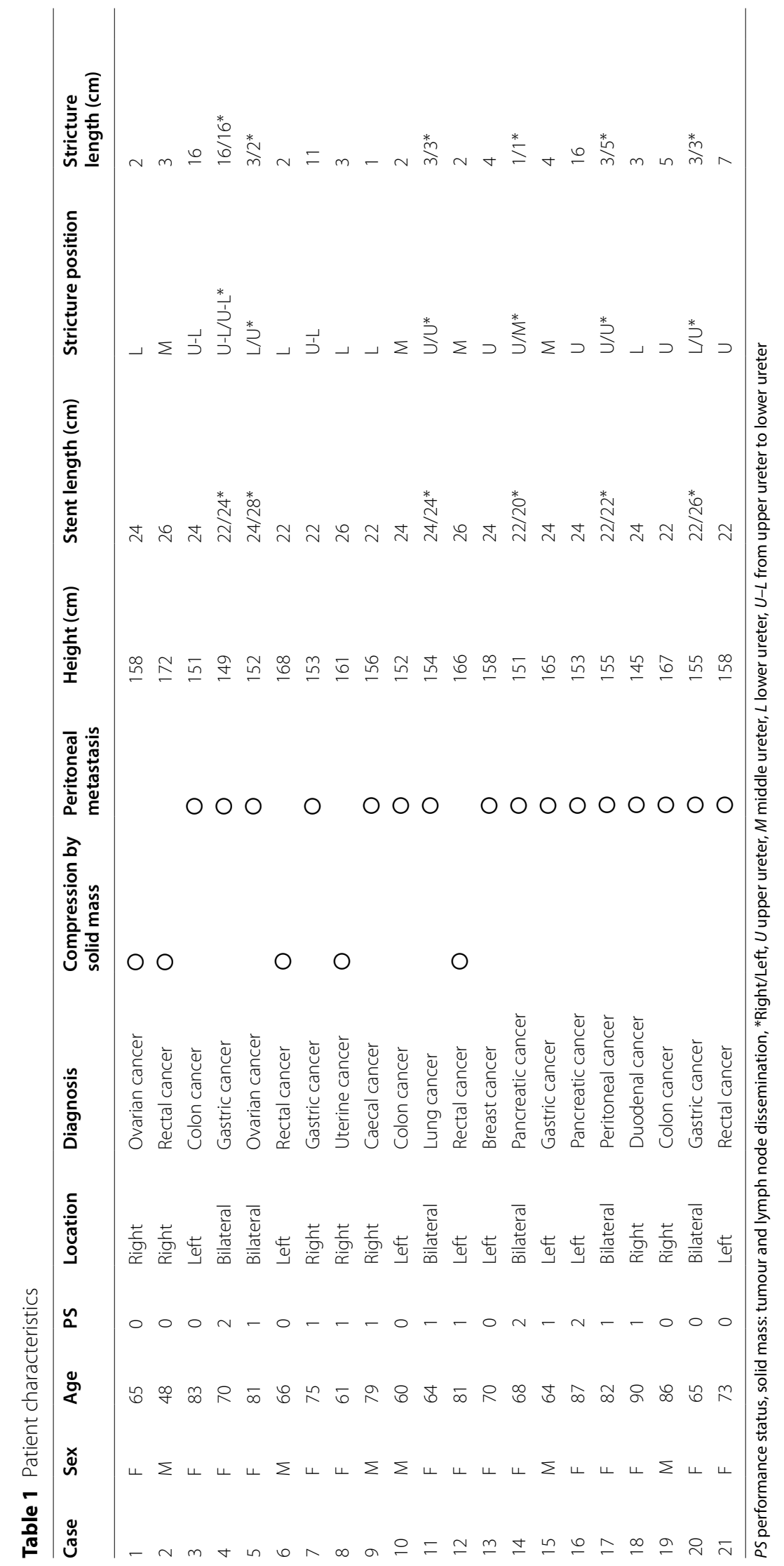




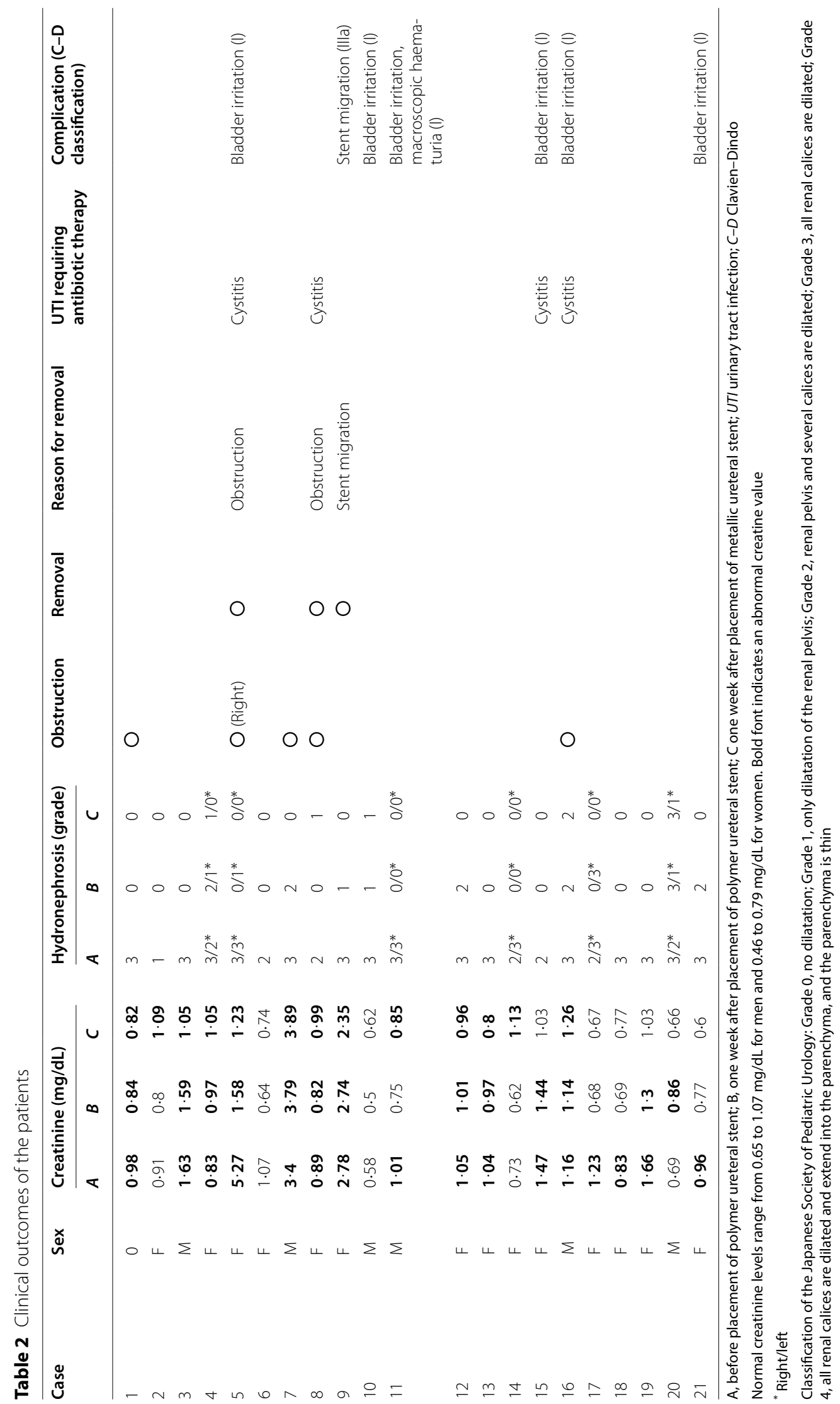




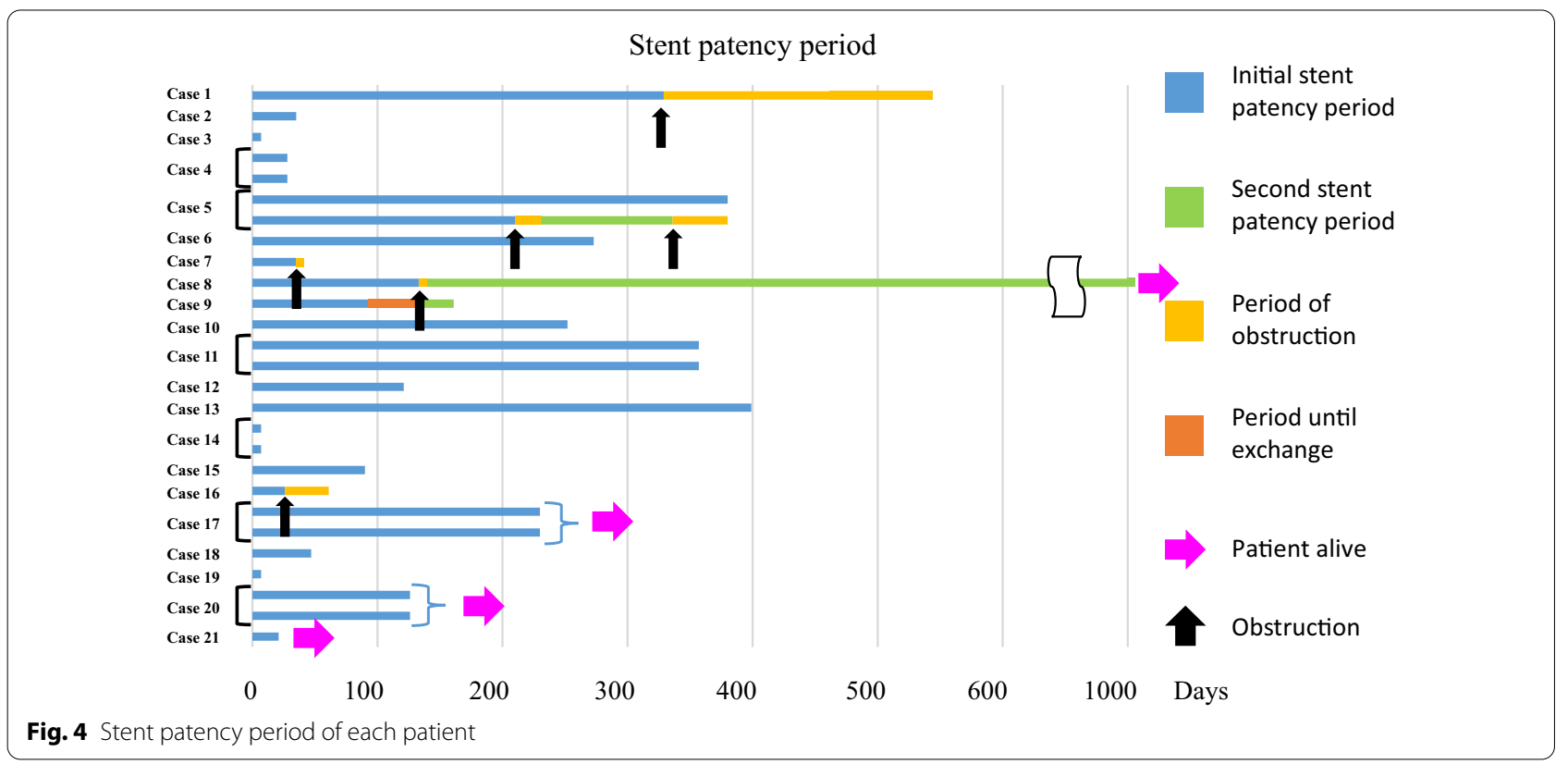

$(\mathrm{P}=0.0336)$ (Fig. 5f). We found no significant differences in the stent patency period between the patients in regard to age ( $<70$ years old or not) (Fig. $5 \mathrm{~g})$, performance status $(0$ or not) (Fig. 5h), and serum creatinine before placement of the polymer ureteral stent $(<2 \mathrm{mg} / \mathrm{dL}$ or not) (Fig. 5i). Due to the small number of cases, we could not conduct Cox proportional multivariate analysis.

\section{Discussion}

The polymer ureteral stent is used when treating patients with MUO because of its familiarity and ease of use [11]. Despite its superiority, the rate of obstruction is $35-45 \%$ in patients with MUO treated with this stent [16]. In contrast, the rate of obstruction with the metallic ureteral stent is relatively low at $15.4-17 \%$ [17] and was $19 \%$ in the present study, similar to that of the previous study.

It is noteworthy that upper urinary flow with a metallic ureteral stent may be slower compared with that with a polymer ureteral stent [18]. Hsu et al. reported that the early patency rate of metallic ureteral stents was $80 \%$ in patients with no prior ordinary stent and $95.3 \%$ in those with prior ordinary stent implantation [19]. They found that prior ordinary stent implantation was associated with the early patency rate of metallic ureteral stents in a univariate analysis [19], and in the present study, all patients maintained early patency of their metallic ureteral stents. As a technical point, Liatsikos et al. reported that they needed to dilate the obstructed ureter with a balloon catheter to insert the metallic ureteral stent in $35 \%$ of their patients [20]. In contrast, we could insert a polymer ureteral stent without the need of a balloon catheter in all patients. Wang et al. reported a $15 \%$ rate of technical failure when inserting a metallic ureteral stent because they did not dilate the obstructed ureter with a balloon catheter [21]. They suggest that in certain cases it may be difficult to initially perform metallic ureteral stent placement. In contrast, Chow et al. reported that they could replace a polymer ureteral stent with a metallic ureteral stent in patients with MUO without difficulty [11]. We also could replace the polymer ureteral stents with metallic ureteral stents in our patients without the need of a balloon catheter. Chow et al. also reported the outcome of MUO patients who received a polymer ureteral stent and subsequently a metallic ureteral stent [11]. Their reported patency periods for the polymer and metallic ureteral stents were 1.7 months and 5.3 months, respectively. All patients in the present study could maintain patency of their polymer ureteral stents one month after placement, and the median patency period for

(See figure on next page.)

Fig. 5 a Overall survival. b Overall stent patency. c Stent patency in patients with direct compression by solid mass and peritoneal metastasis. $\mathbf{d}$ Stent patency in patients with bilateral and unilateral obstruction. e Stent patency in patients with and without urinary tract infection (UTI). f Stent patency in patients with a normal serum creatinine level one week after placement of the polymer ureteral stent. $\mathbf{g}$ Stent patency by patient age ( $<70$ years old or not). $\mathbf{h}$ Stent patency by performance status ( 0 or not). $\mathbf{i}$ Stent patency by serum creatinine level before placement of the polymer ureteral stent $(<2 \mathrm{mg} / \mathrm{dL}$ or not) 
a

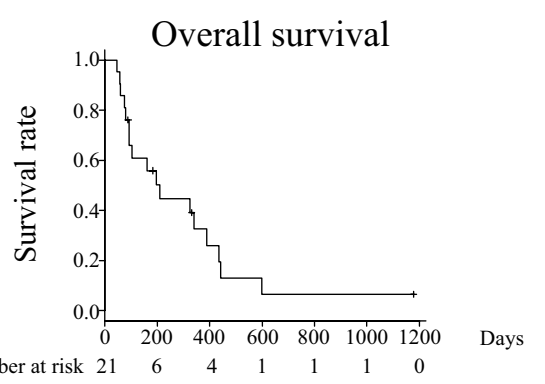

c

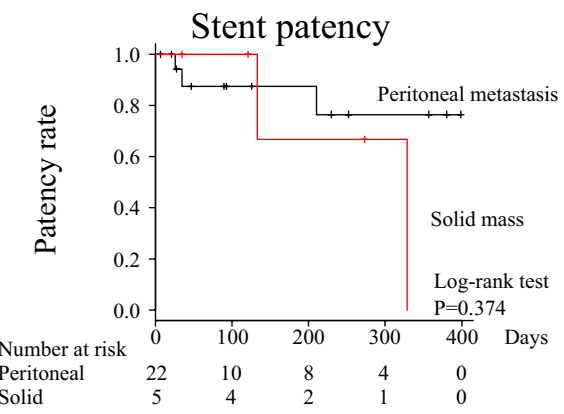

e

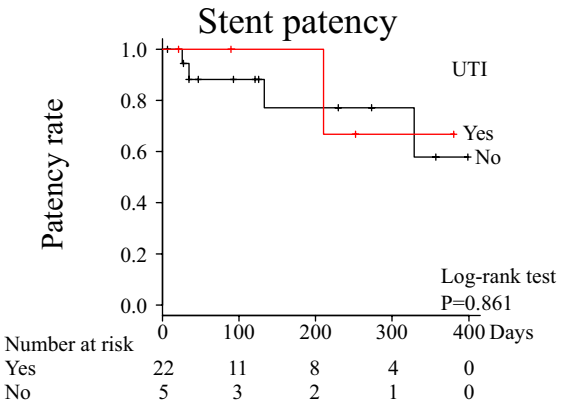

g

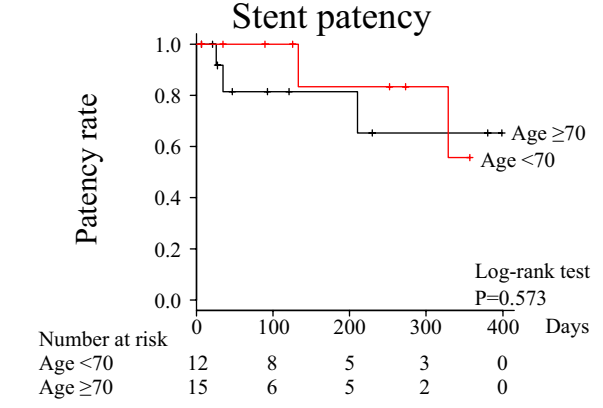

i

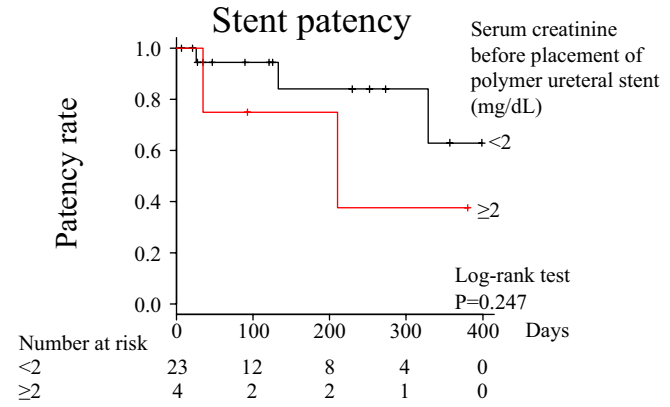

b

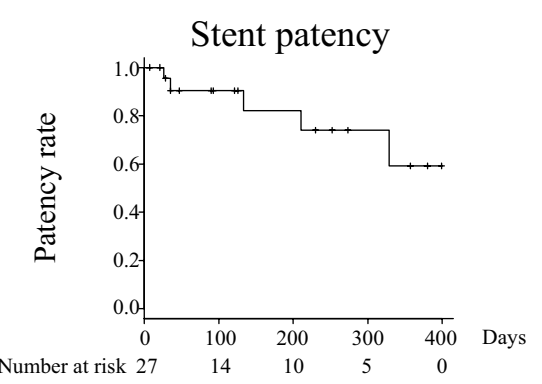

d

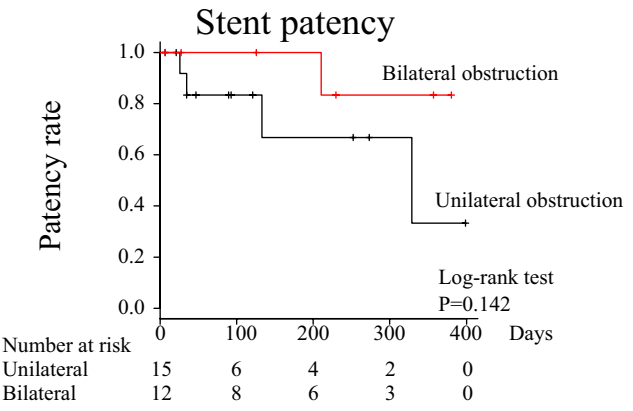

f

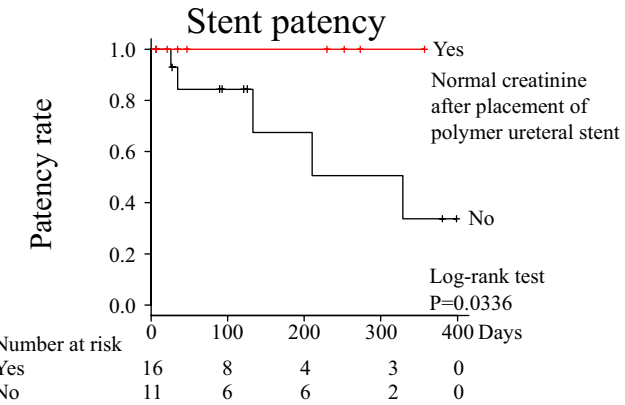

h

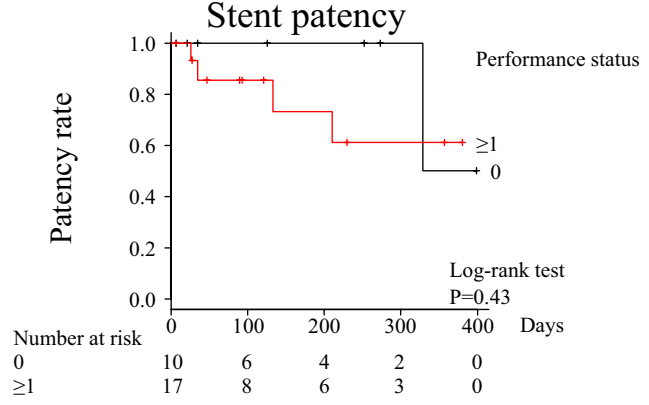

Fig. 5 (See legend on previous page.) 
the metallic ureteral stent was NA (95\% CI 210-NA) due to the inability to extract this value from the Kaplan-Meier curve because the event rate did not reach $50 \%$. Because we wanted to strongly ensure that urinary tract obstruction would be released by polymer ureteral stent placement, which would help not only to maintain renal function but also prevent flank pain and UTI associated with hydronephrosis, we first inserted a polymer ureteral stent and then replaced it with a metallic ureteral stent one month later. We tried to relieve any potential obstruction of the polymer ureteral stent at its initial insertion and to maintain long-term patency with the metallic ureteral stent in our patients with MUO.

It is well known that an indwelling ureteral stent including the metallic ureteral stent can potentially cause urinary symptoms such as bladder irritation, haematuria, and UTI [22]. Previous studies suggested that placement of a stent of the appropriate length might reduce stentrelated complications $[9,17,22]$. Brown et al. noted that direct intraoperative measurement of the ureter length was the superior method [22], but in some patients, including those with a tortuous ureter, it is difficult to measure ureter length appropriately at the first retrograde pyelography. We performed repeat retrograde pyelography at placement of the metallic ureteral stent if we thought re-examination to be necessary.

The risk factors affecting the patency period were reported to be older age, preoperative high serum creatinine level, no lower gastrointestinal cancers, abdominal level obstruction, lymphatic metastasis, and bilateral ureteral obstruction $[4,10,11,23]$. In the present study, abnormal serum creatinine one week after placement of the polymer ureteral stent might be a risk factor affecting a shorter patency period. Asakawa et al. investigated the cause of ureteral obstruction but found no significant factors affecting the patency period [4]. We hypothesised that MUO caused by peritoneal metastasis rather than a solid mass would have a shorter patency period because it disturbed a wide range of ureteral peristaltic movement. However, we found no significant difference in the patency period between a solid mass and peritoneal metastasis. Of note, however, we did find a difference in the patency period between patients with and without a normal serum creatinine one week after placement of the polymer ureteral stent. Although this result was only obtained by univariate analysis, a normal serum creatinine after polymer ureteral stent placement might predict the success of therapy because the polymer ureteral stent releases upper urinary tract obstruction and helps to prevent postrenal problems and the resonance stent maintains this condition, indicating that both are fulfilling their function as stents. Although further studies are required to prove the result with Cox proportional multivariate analysis, we think that the present results may help urologists determine optimal therapy.

All of our patients obtained relief from hydronephrosis one week after placement of the polymer ureteral stent. Patients without a normal serum creatinine one week after polymer ureteral stent placement might also have intrarenal or prerenal causes that were induced by progressive disease and that eventually led to stent obstruction. Many patients with early metallic ureteral stent obstruction were reported to undergo conversion to nephrostomy [24]. We might be able to predict poor outcome by checking the serum creatinine level one week after placement of a polymer ureteral stent.

Generally, our patients with MUO had a short life expectancy, with a median overall survival of 209 days, and this result was similar to that of previous reports [4, 12]. We defined the patency period as the time from the day of initial metallic ureteral stent placement to that of obstruction or last follow-up without obstruction, and not death, because we wanted to ascertain the metallic ureteral stent patency period more precisely. The median patency period was NA ( $95 \%$ CI $210-\mathrm{NA})$, and the oneyear patency rate was $59.2 \%$ (95\% CI 23.2-82.9), which are similar to those of previous studies [24, 25]. Considering the remaining lifetime of patients with MUO and the patency period of metallic ureteral stents, many of these patients will likely not require exchange of their metallic ureteral stent.

Compared with other studies, the present study had two advantages. First, the close follow-up interval allowed more precise ascertainment of the clinical outcome of the patients with MUO. Second, we could select the length of the metallic ureteral stent more accurately, which might have reduced stent-related symptoms.

The present study has some limitations. Our sample size was too small to analyse the factors affecting patency period with Cox proportional multivariate analysis, and thus, the results must be interpreted with caution. Second, we could not compare our study cohort with the standard treatment cohort (insertion of a metallic ureteral stent from the beginning) by randomised study. Third, we did not evaluate QOL with the Ureteric Stent Symptom Questionnaire (USSQ) [26], and we could not use the Japanese version of the USSQ because it was still undergoing validation. Fourth, we did not analyse costeffectiveness. The cost of our method is higher because we initially place a polymer ureteral stent to provide early relief of the obstruction and then more accurately select the length of the metallic ureteral stent to help reduce any stent-related symptoms. 


\section{Conclusion}

The Resonance Metallic Ureteral Stent was effective and safe for treating patients with MUO. A normal serum creatinine one week after placement of the polymer ureteral stent may predict a longer patency period of metallic ureteral stents in patients with MUO.

\section{Abbreviations}

Cl: Confidence interval; MUO: Malignant ureteral obstruction; QOL: Quality of life; USSQ: Ureteric Stent Symptom Questionnaire; UTI: Urinary tract infection.

\section{Acknowledgements}

Not applicable

\section{Authors' contributions}

$\mathrm{YK}, \mathrm{HA}$, and $\mathrm{MH}$ contributed to study conception or the drafting of the manuscript. All authors take responsibility for the data presented therein, and all are aware of, have read, and approve the manuscript being submitted.

\section{Funding}

None.

\section{Availability of data and materials}

The data supporting the findings of this study are presented within the manuscript.

\section{Declarations}

\section{Ethics approval and consent to participate}

The Ethics Committee of the Kinki Central Hospital of Mutual Aid Association of Public School Teachers approved this prospective study on 18 January 2016 (approval no. 276), 27 October 2018 (approval no. 364), and 18 June 2021 (approval no. 421). This study complied with the international ethical recommendations of the Declaration of Helsinki. Written informed consent was obtained from all patients studied.

\section{Consent for publication}

Not applicable.

\section{Competing interests}

The authors declare that they have no competing interests.

Received: 11 May 2021 Accepted: 29 August 2021

Published online: 08 September 2021

\section{References}

1. Kouba E, Wallen EM, Pruthi RS (2008) Management of ureteral obstruction due to advanced malignancy: optimizing therapeutic and palliative outcomes. J Urol 180:444-450. https://doi.org/10.1016/j.juro.2008.04.008

2. Jeong IG, Han KS, Joung JY, Seo HK, Chung J (2007) The outcome with ureteric stents for managing non-urological malignant ureteric obstruction. BJU Int 100:1288-1291. https://doi.org/10.1111/j.1464-410X.2007.07172.x

3. Wong LM, Cleeve LK, Milner AD, Pitman AG (2007) Malignant ureteral obstruction: outcomes after intervention. Have things changed? J Uro 178:178-183. https://doi.org/10.1016/j.juro.2007.03.026

4. Asakawa J, Iguchi T, Tamada S, Ninomiya N, Kato M, Yamasaki T et al (2018) Outcomes of indwelling metallic stents for malignant extrinsic ureteral obstruction. Int J Urol 25:258-262. https://doi.org/10.1111/iju.13500

5. Chung SY, Stein RJ, Landsittel D, Davies BJ, Cuellar DC, Hrebinko RL et al (2004) 15-year experience with the management of extrinsic ureteral obstruction with indwelling ureteral stents. J Urol 172:592-595. https://doi. org/10.1097/01.ju.0000130510.28768.f5
6. Wolf JS Jr (2008) The undoing of ureteral obstruction from malignancy-who and how? J Urol 180:435-436. https://doi.org/10.1016/j.juro.2008.05.037

7. Aravantinos E, Anagnostou T, Karatzas AD, Papakonstantinou W, Samarinas M, Melekos MD (2007) Percutaneous nephrostomy in patients with tumors of advanced stage: treatment dilemmas and impact on clinical course and quality of life. J Endourol 21:1297-1302. https://doi.org/10.1089/end.2006. 0104

8. López-Huertas HL, Polcari AJ, Acosta-Miranda A, Turk TM (2010) Metallic ureteral stents: a cost-effective method of managing benign upper tract obstruction. J Endourol 24:483-485. https://doi.org/10.1089/end.2009.0192

9. Nagele U, Kuczyk MA, Horstmann M, Hennenlotter J, Sievert KD, Schilling D et al (2008) Initial clinical experience with full-length metal ureteral stents for obstructive ureteral stenosis. World J Urol 26:257-262. https://doi.org/10. 1007/s00345-008-0245-4

10. Chow PM, Hsu JS, Huang CY, Wang SM, Lee YJ, Huang KH et al (2014) Metallic ureteral stents in malignant ureteral obstruction: clinical factors predicting stent failure. J Endourol 28:729-734. https://doi.org/10.1089/end. 2013.0792

11. Chow PM, Chiang IN, Chen CY, Huang KH, Hsu JS, Wang SM et al (2015) Malignant ureteral obstruction: functional duration of metallic versus polymeric ureteral stents. PLOS ONE 10:e0135566. https://doi.org/10.1371/journ al.pone.0135566

12. Miyazaki J, Onozawa M, Takahashi S, Maekawa Y, Yasuda M, Wada Ket al (2019) The resonance ${ }^{\circledR}$ metallic ureteral stent in the treatment of malignant ureteral obstruction: a prospective observational study. BMC Urol 19:137. https://doi.org/10.1186/s12894-019-0569-y

13. Williams JR (2005) The ethics activities of the World Medical Association. Sci Eng Ethics 11:7-12. https://doi.org/10.1007/s11948-005-0050-z

14. Shimada K, Kakizaki H, Kubota M, Taki M, Takeuchi H, Hiramatsu Y et al (2004) Standard method for diagnosing dilatation of the renal pelvis and ureter discovered in the fetus, neonate or infant. Int J Urol 11:129-132. https://doi. org/10.1111/j.1442-2042.2003.00755.x

15. Kawahara T, Ito H, Terao H, Yoshida M, Ogawa T, Uemura H et al (2012) Choosing an appropriate length of loop type ureteral stent using direct ureteral length measurement. Urol Int 88:48-53

16. Goldsmith ZG, Wang AJ, Bañez LL, Lipkin ME, Ferrandino MN, Preminger GM et al (2012) Outcomes of metallic stents for malignant ureteral obstruction. J Urol 188:851-855. https://doi.org/10.1016/j.juro.2012.04.113

17. Benson AD, Taylor ER, Schwartz BF (2011) Metal ureteral stent for benign and malignant ureteral obstruction. J Urol 185:2217-2222. https://doi.org/ 10.1016/j.juro.2011.02.008

18. Kallidonis PS, Georgiopoulos IS, Kyriazis ID, Kontogiannis S, Al-Aown AM, Liatsikos EN (2015) The full metallic double-pigtail ureteral stent: review of the clinical outcome and current status. Indian J Urol 31:8-14. https://doi. org/10.4103/0970-1591.134232

19. Hsu JS, Huang CY, Liu KL, Chow PM (2018) Risk factors for primary failure of metallic ureteral stents: experience from a tertiary center. J Endourol. https://doi.org/10.1089/end.2017.0611

20. Liatsikos E, Kallidonis P, Kyriazis I, Constantinidis C, Hendlin K, Stolzenburg JU et al (2010) Ureteral obstruction: is the full metallic double-pigtail stent the way to go? Eur Urol 57:480-486. https://doi.org/10.1016/j.eururo.2009.02. 004

21. Wang HJ, Lee TY, Luo HL, Chen CH, Shen YC, Chuang YC et al (2011) Application of resonance metallic stents for ureteral obstruction. BJU Int 108:428-432. https://doi.org/10.1111/j.1464-410X.2010.09842.x

22. Brown JA, Powell CL, Carlson KR (2010) Metallic full-length ureteral stents: does urinary tract infection cause obstruction? Sci World J 10:1566-1573. https://doi.org/10.1100/tsw.2010.162

23. Chow PM, Hsu JS, Wang SM, Yu HJ, Pu YS, Liu KL (2014) Metallic ureteral stents in malignant ureteral obstruction: short-term results and radiological features predicting stent failure in patients with non-urological malignancies. World J Urol 32:729-736. https://doi.org/10.1007/s00345-013-1143-y

24. Kadlec AO, Ellimoottil CS, Greco KA, Turk TM (2013) Five-year experience with metallic stents for chronic ureteral obstruction. J Urol 190:937-941. https://doi.org/10.1016/j.juro.2013.02.070

25. Patel C, Loughran D, Jones R, Abdulmajed M, Shergill I (2017) The resonance ${ }^{\circledR}$ metallic ureteric stent in the treatment of chronic ureteric obstruction: a safety and efficacy analysis from a contemporary clinical series. BMC Urol 17:16 
26. Joshi HB, Newns N, Stainthorpe A, MacDonagh RP, Keeley FX Jr, Timoney AG (2003) Ureteral stent symptom questionnaire: development and validation of a multidimensional quality of life measure. J Urol 169:1060-1064. https:// doi.org/10.1097/01.ju.0000049198.53424.1d

\section{Publisher's Note}

Springer Nature remains neutral with regard to jurisdictional claims in pub-

lished maps and institutional affiliations.
Submit your manuscript to a SpringerOpen ${ }^{\odot}$ journal and benefit from:

- Convenient online submission

- Rigorous peer review

- Open access: articles freely available online

- High visibility within the field

- Retaining the copyright to your article

Submit your next manuscript at $\boldsymbol{\nabla}$ springeropen.com 